\title{
Exoplanet yield predictions for the future LIFE mission
}

\section{Other Conference Item}

\section{Author(s):}

Kammerer, Jens; Dannert, Felix; Quanz, Sascha Patrick (D); Angerhausen, Daniel; LIFE Collaboration

Publication date:

2021-09

Permanent link:

https://doi.org/10.3929/ethz-b-000523604

\section{Rights / license:}

Creative Commons Attribution 4.0 International

\section{Originally published in:}

EPSC Abstracts 15, https://doi.org/10.5194/epsc2021-328 


\title{
Exoplanet yield predictions for the future LIFE mission
}

\author{
Jens Kammerer ${ }^{1}$, Felix Dannert ${ }^{2,3}$, Sascha Quanz ${ }^{2,3}$, Daniel Angerhausen ${ }^{2,3}$, and the LIFE \\ Collaboration $^{*}$ \\ ${ }^{1}$ Space Telescope Science Institute, United States of America (jkammerer@stsci.edu) \\ ${ }^{2}$ ETH Zurich, Switzerland \\ ${ }^{3}$ National Center of Competence in Research PlanetS (www.nccrplanets.ch) \\ ${ }^{*}$ A full list of authors appears at the end of the abstract
}

Context: Warm, terrestrial exoplanets represent a key component in the exoplanet population when exploring the diversity of planets and investigating the properties of potentially habitable worlds [1]. Subsequently, the LIFE Collaboration is presenting a mission concept for the Large Interferometer for Exoplanets (LIFE) capable of directly imaging the thermal emission of terrestrial exoplanets in the mid-infrared using the technique of nulling interferometry [2]. Since LIFE will explore the previously disfavored parameter-space of mature planets close to their host stars, it needs to spend 2.5 yrs of its 5 yrs mission on detecting previously inaccessible planets. This so-called search phase will provide the sample of exoplanets from which suitable targets for in-depth follow-up observations will be selected. The scientific success of the mission hinges on its capacity to observe a sufficiently large sample of potentially habitable worlds to allow for the formulation of statistically significant results regarding the existence of life in the Universe. These kinds of results are further needed to constrain exoplanet populations and make results comparable to theoretical predictions.

Aim: We aim to predict the exoplanet yield of the LIFE mission search phase in total numbers of detected planets and properties of the planetary sample. Our predictions are based on the assumption of the measurement principle of LIFE and all pertinent astrophysical sources of noise (stellar leakage, local zodiacal, and exozodiacal dust) degrading the quality of the measurements. While our current simulations only account for random background noise, we aim to include an implementation of instrumental noise sources once a preliminary mission design has been developed.

Methods: The prediction of the search phase yield can be subdivided into three steps. First, a catalog of nearby stars [2] is populated with synthetic planets drawn based on Kepler statistics [3]. For each of these planets, a nulling inteferometric observation with LIFE is simulated under the assumption of the astrophysical noise contributions mentioned above. Lastly, the time available in the search phase is distributed among the targets such that the total number of detected planets is maximised.

Results: We predict that LIFE will be able to detect roughly 230 terrestrial exoplanets within the 2.5 yrs search phase. Figure 1 shows the distribution of this sample in the planet radius and insolation plane. LIFE will be most efficient in finding warm, super-Earth-sized planets (as defined in [4]), but enables detections ranging from $0.5-6 \mathrm{R}_{\square}$ in radius and $10^{-1}-10^{3} \mathrm{~S}_{\square}$. We find that the exoplanet yield is a strong function of the mirror size and that the uncertainties are dominated by uncertainties in 
the underlying planet population.

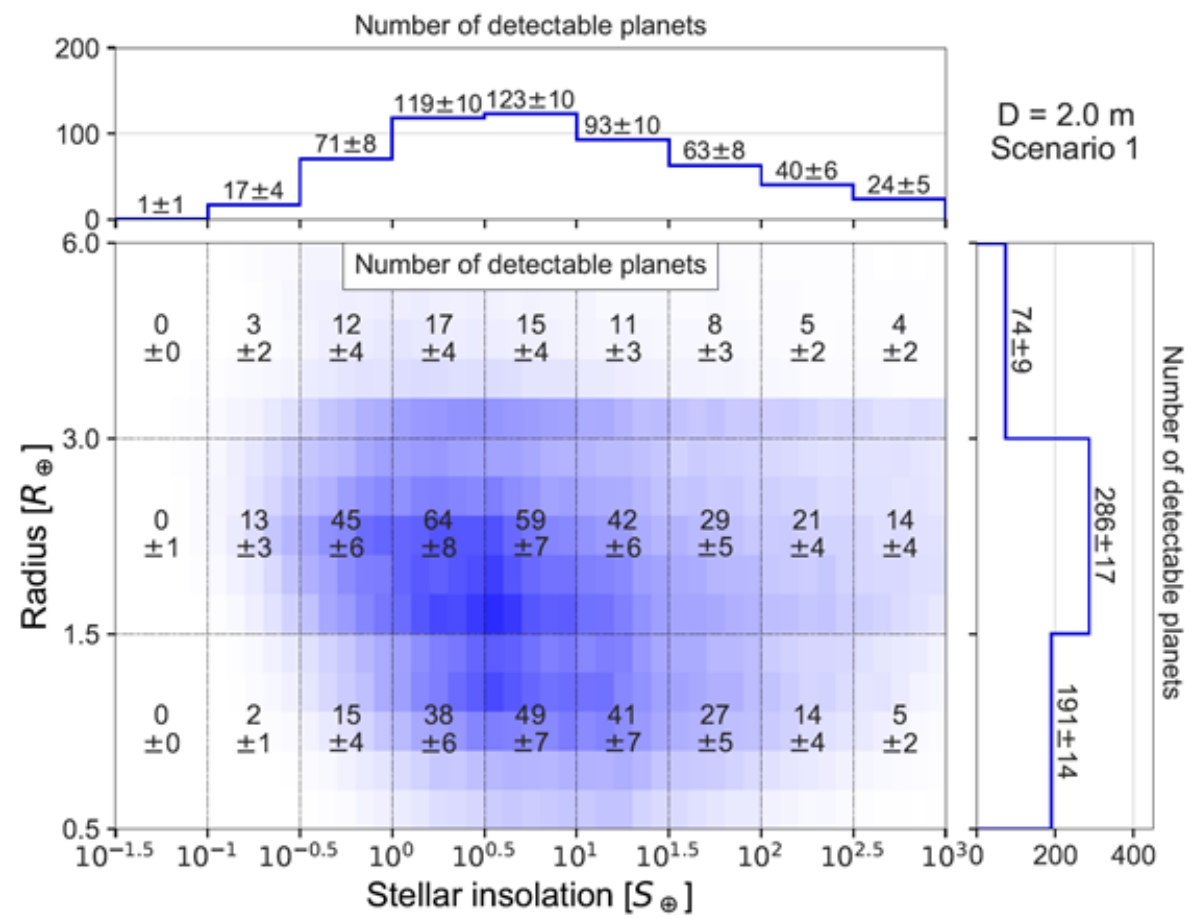

Fig 1: Total detection yield for terrestrial exoplanets in the radius-insolation plane. The $1-\sigma-u n c e r t a i n t y$ is obtained through a Monte-Carlo approach in the planet population. (LIFE Collaboration et al. 2021)

We show that the properties of the exoplanet sample do not only depend on the characteristics of the instrument, but furthermore on the distribution of the available observing time among stellar targets. Figure 2 demonstrates that a distribution optimized towards detecting planets in the habitable zone of their parent star (scenario 2) can increase the total number of these potentially habitable planets by $\sim 60 \%$. However, such optimizations come at the cost of a reduced number of detections in other planet categories. 

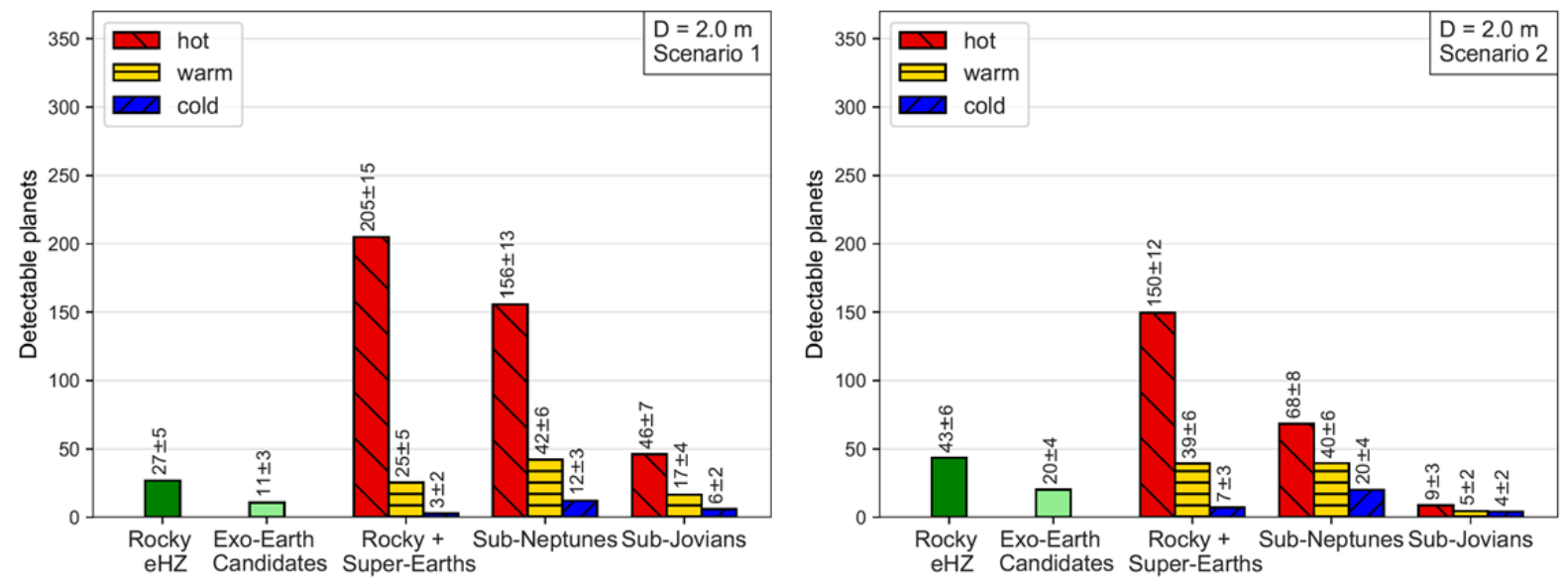

Fig 2: Total exoplanet detection yield using a planet classification scheme. The bars show the number of expected planet detections including the statistical 1- $\sigma$-uncertainty from the Monte Carlo approach. Left panel: scenario 1, i.e., the search phase is optimized for maximizing the total number of exoplanets. Right panel: scenario 2, i.e., the search phase is optimized for maximizing the number of rocky eHZ exoplanets. (LIFE Collaboration et al. 2021)

Depending on the optimization scenario, 27-43 of the detected planets will reside in the empirical habitable zone [5] of their host stars. This is thought to be a sufficient number of planets to effectively constrain the ratio of terrestrial planets in the habitable zone which provide conditions for liquid water to exist on their surface [6]. Since a significant fraction of these planets will be around M-type stars, a discussion of the habitability potential in those conditions [7] needs to be revised with the help of JWST observations.

The value that the LIFE mission will add to the sample of known exoplanets is significant. Figure 3 demonstrates how the detection capability of LIFE for terrestrial exoplanets reaches to significantly smaller planets, covering the region in parameters space occupied by the four terrestrial planets in the Solar System. We are able to demonstrate that in terms of number of detections, LIFE will provide a potential similar to that of the LUVOIR concept and superior to that of the HabEx concept. Lastly, we raise the following discussion point: We have shown that the number of predicted detections depends not only on the instrument performance, but also on the underlying synthetic exoplanet sample and the distribution of the observing time. Since the assumptions for the latter two points likely deviate between mission concepts, we reiterate a performance measure which can decouple and display the instrument performance. 


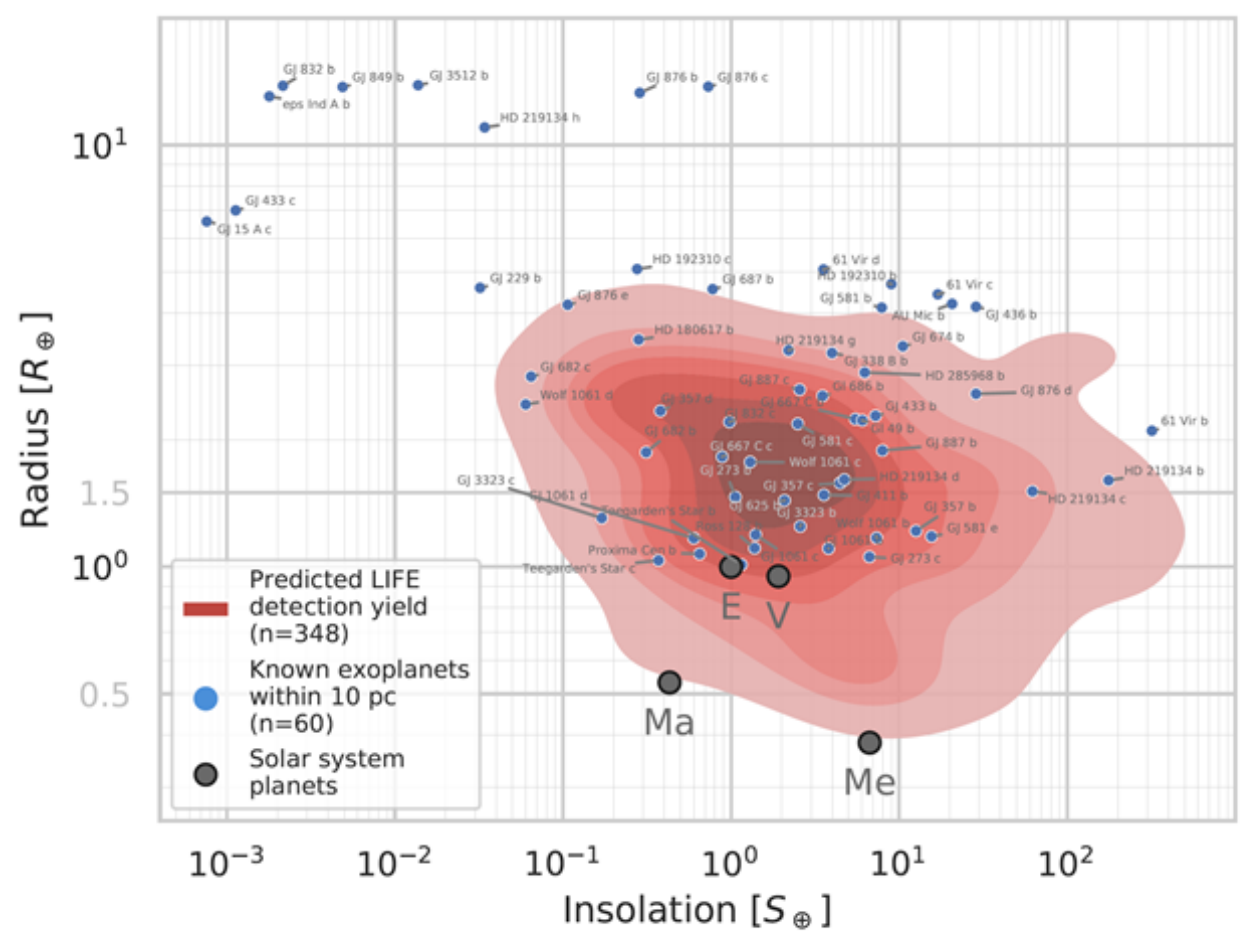

Fig 3: Comparison of exoplanet detections with LIFE to known Solar System planets and exoplanets. The LIFE yield for the reference case (scenario 2) is shown in red contours using a kernel density estimate of the detected sample. Every shaded contour level corresponds to 50 exoplanets detected in the respective parameter space. Blue points represent 60 out of 79 known exoplanets within $10 \mathrm{pc}$ of the Sun for which we could estimate their radius and insolation level. Grey points represent the four rocky planets in the Solar System ( $\mathrm{E}=\mathrm{Earth}, \mathrm{V}=$ Venus, Ma=Mars, Me=Mercury). (LIFE Collaboration et al. 2021)

\section{References:}

[1] Committee on Exoplanet Science Strategy: NASA Exoplanet Science Strategy, The National Academy of Sciences, 2018

[2] LIFE Collaboration et al.: Large Interferometer For Exoplanets (LIFE): I. Improved exoplanet detection yield estimates for a large mid-infrared space-interferometer mission, arXiv, 2021

[3] Kammerer, Jens, and Quanz, Sascha P.: Simulating the Exoplanet Yield of a Space-Based MidInfrared Interferometer Based on Kepler Statistics, Astronomy and Astrophysics, Vol. 609, 2018

[4] Kopparapu, Ravi Kumar, et al.: Exoplanet Classification and Yield Estimates for Direct Imaging Missions, The Astrophysical Journal, Vol. 856, 2018

[5] Kopparapu, Ravi Kumar, et al.: Habitable Zones around Main-Sequence Stars: Dependence on Planetary Mass, The Astrophysical Journal Letters, Vol. 787, 2014

[6] Quanz, Sascha P., et al.: Atmospheric Characterization of Terrestrial Exoplanets in the MidInfrared: Biosignatures, Habitability \& Diversity, ESA Voyage 2050 White Paper, arXiv, 2019

[7] Shields, Aomawa L., et al.: The habitability of planets orbiting M-dwarf stars, Physics Reports, Vol. 663, 2016

LIFE Collaboration: https://www.life-space-mission.com/ 